\title{
Effervescent atomization spray: Understanding the modeling process
}

Understanding the atomization spray process is not only of academic interest but also is important to various industry applications. Significant experimental investigations concerning spray behavior have been conducted in the past. Fundamental understanding of the spray process is, however, still lacking and lags behind applications due to the high complexity of its stochastic behavior. Prof. LIN Jianzhong and his group proposed a comprehensive three-dimensional model to predict the droplet mean size and other spray characteristics by describing both primary and secondary atomization. Recently, they reviewed the theories, practical modeling treatments and the main achievements of modeling on effervescent atomization. Their work, entitled "Modeling on effervescent atomization: A review", was published in Sci China Phys Mech Astron, 2011, 54: 2109-2129.

Effervescent atomization is a method involving a twin-fluid process that involves bubbling gas within a liquid. Compared to conventional pressure, rotary and twin-fluid atomizers, the effervescent atomizer offers advantages of smaller drop sizes, reduced injection pressure, lower gas flow, a larger exit orifice, and tolerance for high viscosities. Effervescent atomization has been widely used in gas turbine combustors, IC engines furnaces and boilers, incineration, spray deposition, powder formation and other applications. Recently, it has been successfully applied in pharmaceutical coating and suspension plasma spray. Because effervescent atomization can handle a variety of liquids, it can play a pivotal role in reducing energy consumption during combustion and has potential for broad applications in the manufacture of high quality materials.

Lin and his co-workers from Jiliang University and Zhejiang Universities, China, have devoted their efforts to establish a comprehensive numerical model to explain the phenomena involved in effervescent atomization spray. Their studies cover modeling of liquid fragmentation, the parametric study of various operating conditions, and the development of a fitting formula for droplet mean size and impinging factors.

The effervescent atomization process involves complex fluid-dynamic and transport phenomena. A typical effervescent atomization spray can be divided into four sub-domains. The first sub-domain is internal-mixing atomization, in which atomizing gas is bubbled into the liquid. The second step is a resulting two-phase mixture that is discharged from the atomizer orifice, where the rapidly expanding gas phase will shatter the liquid into fine droplets, which can be referred to as primary atomization. The third domain lies downstream of the spray. The droplets produced by primary atomization are unstable in the turbulent spray and undergo a series of events such as collision, breakup and coalescence, and finally the droplets entrained in the gas jet will impinge on the plate or undergo further mass and heat transfer. The review paper focused on the Lagrangian treatment and introduced a comprehensive model capable of describing both primary and secondary breakup processes and correlating the droplet mean diameter and other spray characteristics with first principle operating conditions. The model comprises two sub-models. The first sub-model is used for simulating the primary breakup of the annular liquid sheath near the orifice and calculating the atomized droplet size. The second sub-model is based on a hybrid Eulerian/Lagrangian coordinate system to simulate the turbulent gas jet and injected droplets. This latter sub-model describes the gas jet and the droplet trajectory in three-dimensional geometry and also considers droplet breakup and collision.

The paper reviews the mechanism of droplet events and the numerical treatments of effervescent atomization, which involved the primary atomization of a Newtonian and a non-Newtonian fluid, particle tracking, secondary atomization and droplets collision. A comprehensive three-dimensional model of droplet-gas flow is introduced to describe the evolution of spray in the effervescent atomization spray. The evolution of droplet mean diameter along the axial distance, the mean size and statistical distribution of atomized droplets at cross sections, as well as the change in droplet velocity are calculated and analyzed to reveal their inner driving forces. The influence of operating conditions and liquid physical properties on atomization performance are discussed. The factors that promote the achievement of good atomization effects are identified. Based on the extensive computation, the influences of various operating conditions and liquid physical properties on atomization effects are quantified. The expressions for the Weber number and the $K$ number, which is related to the operating parameters and liquid properties, are deduced. The formula can be used conveniently and effectively to judge the deposition behaviors of droplets onto surfaces for various Newton liquids. In addition to these achievements, the challenges for future numerical research and the scope for further applications are outlined.

Through academic study and modeling, the critical mechanisms and important parameters involved in effervescent atomization have been deeply understood and the applications of effervescent atomization technology have been successfully extended. The development of modeling approaches to atomization spray is based on previous studies involving many researchers from various institutions and universities.

This work was supported by the National Natural Science Foundation of China (11132008 and 11002136).

See the article: Qian L J, Lin J Z. Modeling on effervescent atomization: A review. Sci China Phys Mech Astron, 2011, 54: 2109-2129

Open Access This article is distributed under the terms of the Creative Commons Attribution License which permits any use, distribution, and reproduction in any medium, provided the original author(s) and source are credited. 